\title{
Evidence for peripheral and central processes in taste adaptation
}

\author{
DOUGLAS J. GILLAN \\ General Foods Technical Center, Tarrytown, New York
}

\begin{abstract}
Adaptation is one of the fundamental phenomena of taste perception. Adaptation is usually measured, as it was here, as the perceived intensity of a test stimulus following prolonged stimulation by an adapting stimulus. In this experiment, both the adapting and test stimuli were delivered via two pieces of filter paper-one soaked in sucrose, the other in water-placed on opposite sides of the tongue. Adaptation was greatest when the spatial relations of sucrose and water were the same for both the adapting and test stimuli, but was significant in comparison with a no-adaptation control when the spatial relations of the adapting and test stimuli were different. The result is discussed in terms of peripheral and central processes in taste adaptation: Peripheral processes were assumed to be engaged only when sucrose had the same spatial location for the adapting and test stimuli, whereas central processes were assumed to be responsible for the adaptation observed when sucrose had a different spatial location for the adapting and test stimuli. The implications of a multiprocess view of adaptation for psychophysical models of taste adaptation, the use of adaptation as a research tool, and future research on adaptation are discussed.
\end{abstract}

Adaptation is one of the central phenomena in taste perception (see recent reviews by Bartoshuk, 1978 , and McBurney, 1978). Adaptation in the taste system, as in other sensory systems, has been defined as a decrease in sensation with prolonged stimulation. Much of the research on taste adaptation has focused on the empirical and parametric description of the phenomenon, investigating the time course and completeness of adaptation (e.g., Gent, 1979; Gent \& McBurney, 1978; Meiselman, 1968), the effects of adaptation on detection, recognition, and difference thresholds (O'Mahoney, 1979; McBurney, Kasschau, \& Bogart, 1967), and cross-adaptation and the taste of water following adaptation (Bartoshuk, 1968; McBurney \& Bartoshuk, 1973; McBurney, Smith, \& Schick, 1972; Schiffman, Cahn, \& Lindley, 1981; Smith \& McBurney, 1969).

Psychophysicists have generally assumed that taste adaptation is due to a single peripheral process. For example, Gent (1979) designed her research "to learn whether the rate of adaptation, which appears to be a peripheral process, is characteristic of the particular nerve involved or if it is characteristic of the gustatory system in general" (p. 304) (see also Gent \& McBurney, 1978; Lawless, 1979). This single-process view of adaptation arises from physiological research on the decline in peripheral nerve activity with pro-

This research was supported by the General Foods Technical Center. The author thanks Adrienne Mikovits for her technical assistance and the reviewers for helpful comments. Requests for reprints should be sent to the author at the General Foods Technical Center, T23-1, 555 South Broadway, Tarrytown, NY 10591. longed taste stimulation (Diamant \& Zotterman, 1969; Sato, 1971). For example, Diamant and Zotterman observed that the decrease across time in the activity of the chorda tympani to a tastant continuously applied to the tongue paralleled the decrease in the subject's sensation; consequently, they concluded that "there is no need to postulate the existence of central adaptation mechanisms" (p. 434). There are two relevant points concerning the physiological data. First, adaptation is a psychological phenomenon; physiological mechanisms and psychological phenomena and processes are not necessarily isomorphic. Second, even if one accepts that peripheral physiological mechanisms play a role in the psychological phenomenon of taste adaptation, that doesn't preclude the possibility that central processes might also be involved. That possibility had not been empirically addressed until the research described in this report.

The purpose of the present research was to investigate the possible influence of peripheral and central processes on taste adaptation. The logic of the experiment was as follows: If taste adaptation is sensitive to a peripheral manipulation, then a peripheral process is involved in adaptation. The peripheral manipulation used here was the spatial relation on the tongue of an adapting stimulus and a subsequent test stimulus. In addition, if there is still significant adaptation when the involvement of peripheral processes is prevented, then central processes must also play a role in adaptation. The involvement of peripheral processes was prevented on some trials in this experiment by having the adapting and test stimuli 
occur at different spatial locations on the tongue. Similar logic has been used to study peripheral and central processes in mixture suppression (Gillan, 1982, 1983b).

\section{Method}

\section{Subjects and Stimuli}

Seven General Foods employees volunteered to participate; none of the subjects were specially trained tasters, but all had had previous experience in psychophysical experiments. The adapting stimuli were delivered by means of $15 \times 15 \mathrm{~mm}$ squares of filter paper (Whatman brand, ashless No. 41); the test stimuli were delivered via $10 \times 10 \mathrm{~mm}$ filter paper squares (see Collings, 1974, Collings, Lindberg, \& McBurney, 1976, Gent, 1979, Gent \& McBurney, 1978, and Gillan, 1982, for other taste research using filter paper to deliver tastants). The taste solution on every trial was 1.2 M sucrose. (Preliminary research had indicated that $1.2 \mathrm{M}$ sucrose on a $15 \times 15 \mathrm{~mm}$ piece of filter paper was recognizably, but not extremely, sweet.) The subjects also received filtered tap water as a stimulus.

\section{Procedure}

In each of three sessions, the subjects received six different trials. The general procedure on each trial was as follows: First, a subject rinsed his or her mouth thoroughly with water. Next, the experimenter used two pairs of forceps to pick up two $15 \times 15 \mathrm{~mm}$ pieces of filter paper, soaked the filter papers in the sucrose solution and/or water, touched them to the sides of the cups containing the solutions, and shook them gently 1.2 times to remove excess fluid. Then the experimenter simultaneously placed the two pieces of filter paper on the front of the subject's extended tongue equidistant from the midline, with $10-15 \mathrm{~mm}$ separating them. These pieces of filter paper served as the adapting stimulus and stayed on the subject's extended tongue for $40 \mathrm{sec}$.

During the 40-sec adaptation period, the experimenter soaked one $10 \times 10 \mathrm{~mm}$ piece of filter paper in $1.2 \mathrm{M}$ sucrose and a second in water. These pieces of filter paper served as the test stimulus. The excess fluid was removed and the pieces of filter paper were placed on a clean glass surface separated by at least $50 \mathrm{~mm}$. At the end of $40 \mathrm{sec}$, the experimenter removed the adapting stimulus filter papers from the subject's tongue, then quickly picked up the test stimulus papers and placed them on the subject's tongue. The areas on the tongue for the test stimulus papers were inside the areas on which the larger adapting stimulus papers had sat. The time required to remove the adapting stimulus papers and replace them with test stimulus papers was kept to the minimum and ranged from 2 to $4 \mathrm{sec}$. The subject remoyed the test stimulus papers after making his or her intensity judgment, generally within 5 to 10 sec.

There were three basic types of trials-control, adaptationsame, and adaptation-different. For the adapting stimulus on control trials, both pieces of filter paper were soaked in water; on adaptation trials, the adapting stimulus consisted of one piece of filter paper soaked in $1.2 \mathrm{M}$ sucrose and another soaked in water. The test stimulus on all trials was a piece of filter paper soaked in 1.2 $\mathrm{M}$ sucrose and another soaked in water. $\mathrm{On}$ adaptation-same trials, the right-left tongue location of the sucrose-soaked filter paper was the same for both the adapting and test stimuli. In other words, there were two different adaptation-same trials, WS $\rightarrow$ WS and $S W \rightarrow S W$, where the spatial relations of the letters correspond to the spatial relations of sucrose and water on the tongue. On adaptation-different trials, the right-left location of the adapting and test stimuli differed; those trials can be represented as $W S \rightarrow S W$ and $S W \rightarrow W S$. Likewise, there were two control trials$W W \rightarrow S W$ and $W W \rightarrow W S$. Each subject's random order for the six trials was different in each of the three sessions and was different from the other subjects'. The interval between trials was approximately 2 min.
The subjects judged the intensity of only the test stimulus on every trial using magnitude estimation (e.g., Stevens, 1975). In addition, at the start of every session, each subject received a standard stimulus consisting of two $15 \times 15 \mathrm{~mm}$ pieces of filter paper, one soaked in 1.2 M sucrose, the other soaked in water, placed 10 . $15 \mathrm{~mm}$ apart on the front of the tongue and equidistant from the midline. The subjects were instructed to consider the standard to have a sweetness of 100 and to judge the test stimulus on all other trials in relation to it.

\section{Results and Discussion}

The measure of adaptation in this experiment was the sweetness of the test stimulus: the lower its sweetness, the greater the adaptation. The logic of the experiment assumes that peripheral adaptation processes could be engaged only on adaptation-same trials, but central adaptation processes could be engaged on both adaptation-same and adaptationdifferent trials. Consequently, peripheral adaptation processes should lead to lower judgments of the test stimulus sweetness on adaptation-same than on adaptation-different trials, whereas central processes should produce lower judgments on adaptationdifferent than on control trials. The results, shown in Table 1, display this pattern. Sweetness judgments differed as a function of trial type $[F(2,12)=23.0$, $\mathrm{p}<.001$ ]. A Newman-Keuls test (e.g., Keppel, 1973) showed that the judgments of test stimulus sweetness were lowest on adaptation-same trials and lower on adaptation-different than on control trials (all ps $<.01$ ).

Although significant adaptation on adaptationdifferent trials is consistent with the hypothesis that central processes play a role in adaptation, two alternative hypotheses should be considered. One alternative is that if sucrose diffused from the sucrosesoaked filter paper across the tongue during the 40-sec adaptation period, then adaptation on adaptation-different trials might have a peripheral mechanism. However, chemical analysis by gas chromatograph of the filter paper on the side of the tongue opposite to the sucrose-soaked filter paper indicates that no measurable sucrose (or the products of its breakdown in the mouth, glucose and fructose) diffused across the tongue: The gas chromatograph, which could detect any sucrose, glucose, or fructose above a level of 10 parts per million (approximately $.00003 \mathrm{M}$ sucrose), detected none of these sugars on the opposite-side filter paper. Therefore, it appears

Table 1

Mean Sweetness Judgments on Control and Adaptation Trials

\begin{tabular}{lc}
\hline \multicolumn{1}{c}{ Trial Type } & Sweetness \\
\hline Control & 115.1 \\
Adaptation-Different & 75.4 \\
Adaptation-Same & 31.2 \\
\hline
\end{tabular}


that adaptation on adaptation-different trials could not have had a peripheral mechanism.

A second alternative hypothesis suggests that a central process produced the adaptation on adaptationdifferent trials; however, according to this hypothesis that central process would be specific to the conditions on those trials. Specifically, if a subject localized the sweet taste during the adapting stimulus, then, when the sucrose was subsequently presented on the opposite side of the tongue during the test stimulus, he or she would have to shift his or her attention. The low judgments of the test stimulus sweetness on adaptation-different trials might be due to either (1) a delay in processing the sweetness of the test stimulus caused by the time required to shift attention, or (2) disruption in the processing of sweetness caused by the effort involved in shifting attention. Because there is no shift in location in most adaptation procedures, including adaptationsame trials in this experiment, such a central process would not operate generally to produce adaptation. However, an unpublished experiment from this labofatory suggests that people do not readily localize tastes under conditions similar to those used here. In that experiment, each subject had a $1.5 \times 1.5 \mathrm{~cm}$ piece of filter paper on the right side and a similar piece of filter paper on the left side of his or her tongue. One piece was soaked in $.8 \mathrm{M} \mathrm{NaCl}$ and the other in 1.2 $\mathrm{M}$ sucrose. The subjects were asked to identify which side of the tongue contained a sweet tastant and which side contained a salty tastant. Subjects' correct identification of the locations did not differ significantly from chance. If people do not readily locate a taste on the tongue, then it is unlikely that a mechanism based on localization of attention to tastes could have produced the adaptation on adaptation-different trials. Consequently, the central process that produced that adaptation is probably one that operates generally in adaptation procedures.

A simple response, usually a judgment of the intensity of a test stimulus following prolonged exposure to an adapting stimulus, serves as the measure of adaptation in most taste research. Despite the simplicity of that response, the present data and other data from this laboratory (Gillan, 1983a) suggest that there are multiple processes underlying the measure of adaptation. A multiprocess view of adaptation has important implications for psychophysical models of taste adaptation, for the use of adaptation as a research tool, and for future research on adaptation.

First, a multiprocess view of taste adaptation suggested by the above data indicates that adaptation involves at least two stages-an initial, peripheral stage and a subsequent, central stage. That central stage of adaptation may involve such processes as a decrease in the attention to or processing of a persistent stimulus (e.g., Broadbent, 1958; Kahneman, 1973; Treisman, 1969) or habituation (e.g., Thompson \& Spencer,
1966; Wagner, 1976). In addition, there is evidence that central processes also play a role in adaptation in other sense modalities; Cain (1977) observed a significant amount of odor adaptation even when the adapting and test odors were presented to different nostrils.

A multiprocess view of adaptation suggests that adaptation may not be an especially useful tool for studying other taste phenomena. For example, one use to which researchers have put adaptation is in studying mixture suppression (e.g., Bartoshuk, 1979; Bartoshuk \& Seibyl, 1982; Lawless, 1979, 1982a, 1982b). Recent research from this laboratory (Gillan, $1982,1983 \mathrm{~b}$ ) indicates that both peripheral and central processes play a role in mixture suppression, and the present data indicate that adaptation also involves multiple processes. Therefore, it seems likely that any interaction between adaptation and mixture suppression would be complex, and consequently, would be unlikely to clarify the specific mechanisms of either phenomenon.

Finally, a multiprocess approach to adaptation suggests a variety of research questions that need to be addressed. Perhaps most importantly, what are the conditions that activate the peripheral adaptation process and the central adaptation process? The only condition investigated here was continuous stimulation for relatively long periods. A second question concerns the ways in which the processes interact: Do their effects simply add or is their relation more complex? A third important area for investigation will be a precise description of what the processes are. $\mathbf{R e}-$ search on these topics will provide the basis for a more complete psychophysical model of taste adaptation.

\section{REFERENCES}

Bartoshui, L. M.; \& Seibyl, J. P. (1982, April). Suppression of bitterness of $\mathrm{QHCl}$ in mixtures: Possible mechanisms. Paper presented at the meeting of the Association for Chemoreception Sciences, Sarasota, Florida.

Bartoshux, L. M. (1968). Water taste in man. Perception \& Psychophysics, 3, 69-72.

Bartoshur, L. M. (1978). Gustatory system. In R. B. Masterson (Ed.), Handbook of behavioral neurobiology (Vol. 1). New York: Plenum.

BArtoshur, L. M. (1979). Taste interactions in mixtures of sucrose with $\mathrm{NaCl}$ and sucrose with QHCl. Society of Neurosciences Abstracts, 5, 125.

Brondenent, D. E. (1958). Perception and communication. London: Pergamon Press.

CaIn, W. S. (1977). Bilateral interaction in olfaction. Nature, $268,50-52$.

Collings, V. B. (1974). Human taste response as a function of locus of stimulation on the tongue and soft palate. Perception \& Psychophysics, 16, 169-174.

Collings, V. B., LindBerg, L., \& McBurney, D. H. (1976). Spatial interactons of taste stimuli on the human tongue. Perception \& Psychophysics, 19, 69-71.

Diamant, H., \& Zotrerman, Y. (1969). A comparative study of the neural and psychophysical response to taste stimuli. In 
C. Pfaffman (Ed.), Olfaction and taste III. New York: Rockefeller University Press.

Gent, J. F. (1979). An exponential model for adaptation in taste. Sensory Processes, 3, 303-316.

Gent, J. F., \& McBurney, D. H. (1978). Time course of gustatory adaptation. Perception \& Psychophysics, 23, 171-175.

GillAN, D. J. (1982). Mixture suppression: The effect of spatial separation between sucrose and $\mathrm{NaCl}$. Perception \& Psychophysics, 32, 504-510.

Gillan, D. J. (1983a). The relation of the physical intensity and the perceived intensity of the adapting stimulus to taste adaptation. Manuscript submitted for publication.

Gillan, D. J. (1983b). Taste-taste, odor-odor, and taste-odor mixtures: Greater suppression within than between modalities. Perception \& Psychophysics, 33, 183-185.

Kahneman, D. Attention and effort. Englewood Cliffs, NJ: Prentice-Hall.

KePPEL, G. (1973). Design and Analysis: $A$ researcher's handbook. Englewood Cliffs, NJ: Prentice-Hall.

LAwLEss, H. T. (1979). Evidence for neural inhibition in bittersweet taste mixtures. Journal of Comparative and Physiological Psychology, 93, 538-547.

LAwLess, H. (1982a). Adapting efficiency of salt-sucrose mixtures. Perception \& Psychophysics, 32, 419-422.

Lawless, H. (1982b). Paradoxical adaptation to taste mixtures. Physiology \& Behavior, 25, 149-152.

McBurney, D. H. (1978). Psychological dimensions and perceptual analyses of taste. In E. C. Carterette \& M. P. Friedman (Eds.), Handbook of perception (Vol. 6A). New York: Academic Press.

McBurney, D. H., \& Bartoshur, L. M. (1973). Interactions between stimuli with different taste qualities. Physiology \& Behavior, 10, 1101-1106.

McBurney, D. H., Kasschau, R. A., \& Bogart, L. M. (1967).
The effects of adaptation on taste buds. Perception \& Psychophysics, 2, 175-178.

McBurney, D. H., Smith, D. V., \& Shick, T. R. (1972). Gustatory cross-adaptation: Sourness and bitterness. Perception \& Psychophysics, 11, 228-232.

Meiselman, H. L. (1968). Magnitude estimations of the course of gustatory adaptation. Perception \& Psychophysics, 4, 193-196.

O'MAhONEY, M. (1979). Salt taste adaptation: The psychophysical effects of adapting solutions and residual stimuli from prior tastings on the taste of sodium chloride. Perception, 8, 441-476.

Sato, T. (1971). The site of gustatory neural adaptation. Brain Research, 34, 385-388.

Schiffman, S. S., Cahn, H., \& Lindley, M. G. (1981). Multiple receptor sites mediate sweetness: Evidence from cross-adaptation. Pharmacology, Biochemistry, and Behavior, 15, 377-388.

Smith, D. V., \& McBurneY, D. H. (1969). Gustatory crossadaptation: Does a single mechanism code the salty taste? Journal of Experimental Psychology, 80, 101-105.

Stevens, S. S. (1975). Psychophysics. New York: Wiley.

Thompson, R. F., \& Spencer, W. A. (1966). Habituation: A model phenomenon for the study of neuronal substrates of behavior. Psychological Review, 73, 16-43.

Treisman, A. M. (1969). Strategies and models of selective attention. Psychological Review, 76, 282-299.

Wagner, A. R. (1976). Priming in STM: An information processing mechanism for self-generated or retrieval-generated depression in performance. In T. J. Tighe \& R. N. Leaton (Eds.), Habituation: Perspectives for child development, animal behavior, and neurophysiology. Hillsdale, NJ: Erlbaum.

(Manuscript received May 12, 1983;

revision accepted for publication October 16, 1983.) 